\title{
Large-Mass and High-Temperature Behaviour in Perturbative Quantum Field Theory
}

\author{
N. P. Landsman \\ Institute for Theoretical Physics, University of Amsterdam, Valckenierstraat 65, \\ NL-1018 XE Amsterdam, The Netherlands
}

\begin{abstract}
Bounds for large-mass behaviour in renormalized perturbation expansions at zero temperature, which were previously obtained by Manoukian and Caswell-Kennedy in momentum space, are rederived in the parametric representation. A very simple unified proof of the BPHZ theorem and the decoupling theorem is also given. A new technique for asymptotic analysis, based on a generalized Kontorovich-Lebedev integral transform, is introduced. This method is applied to find the leading high-temperature behaviour of perturbative field theories in the imaginary-time formalism. We prove that diagrams containing nonstatic modes, which at high temperature behave like particles with a large mass, are suppressed relative to purely static diagrams. This rigorously proves a limited form of dimensional reduction at infinite temperature.
\end{abstract}

\section{Introduction}

In the early eighties it has been suggested that at very high temperatures field theories in the imaginary-time (Matsubara) formalism would undergo a form of dimensional reduction $[5,14,16]$. The presence of a nonzero temperature $T$ can be incorporated in quantum field theory by compactifying the Euclidean time axis to a circle with radius $\beta=T^{-1}$ [18], and this obviously motivates the idea of a dimensional reduction from $d$ to $d-1$ dimensions.

In perturbation theory in momentum space the temperature enters in the guise of a mass $m=2 \pi n T, n \in Z$, which is present in each (bosonic) propagator [18]. The analogy with the decoupling theorem for heavy particles [4, 2, 20, 9] then suggests that at high temperatures the nonzero modes $(n \neq 0)$ decouple at low momenta, leaving an effective three-dimensional theory, consisting of the zero modes only, behind. In contradistinction to the ordinary decoupling theorem, it is here supposed that an infinite tower of massive particles decouples.

In order to prove that such a dimensional reduction mechanism indeed applies, one ought to state a renormalization scheme in which the nonstatic modes decouple 
[17] (note that the zero-temperature Appelquist-Carazzone theorem [4] heavily depends on the renormalization scheme). A good candidate scheme necessarily involves temperature-dependent subtractions, as one can see by computing oneloop diagrams in the high-temperature limit [17]. The renormalization scheme which turns out to be optimal for dimensional reduction is a finite-temperature generalization of the BPHZ-scheme: one performs zero-momentum subtractions at the actual temperature $T$.

The principal goal of this paper is to derive high-temperature bounds on Green functions renormalized by this scheme, which is also useful outside the context of dimensional reduction. As we shall see, the derivation of these estimates is rather nontrivial, because one has to deal with an infinite number of particles which supposedly decouple. This fact prohibits a direct application of standard techniques for proving such bounds. Indeed, we have found it necessary to use an involved integral transform which thus far has only been used in the study of boundary-value problems [10].

To acquaint the reader with our methods we start by rederiving well-known large-mass estimates in vacuum field theory in Sect. 2. These estimates have been obtained previously by heuristic [9] as well as rigorous [20] momentum-space techniques, which are very hard to generalize to the thermal case. Instead, we rely on the parametric representation $[28,26]$ throughout this paper. We use, in particular, the extremely effective method given by Anikin et al. [3] to incorporate the BPHZ subtractions explicitly, without the need for a forest decomposition or a recursive structure (also cf. [6]). Their technique heavily depends on the use of zeromomentum subtractions (certain additional and superfluous subtractions are made, which have to cancel out in the end), but fortunately it is precisely that scheme which is optimal for the decoupling theorem.

To introduce the notation, as well as some technical results that are needed anyway, we first prove the BPHZ theorem and the decoupling theorem at one stroke, thus simplifying previous proofs of these separate theorems [28, 3, 2]. We then (Sect. 3) derive the known large-mass bounds, firstly employing a Mellin transform à la Bergère-Lam (who employed this technique to obtain largemomentum expansions [7]), and secondly, once again, using a generalized Kontorovich-Lebedev transform. For technical reasons the former integral transform cannot be used to obtain high-temperature expansions, but the latter can; it is complicated enough, however, to justify its introduction in a familiar context.

In Sect. 4 we pass to the finite-temperature case, and state our thermal renormalization scheme. In the course of proving the convergence of our subtraction method we demonstrate a number of technical lemmata which are essential for the high-temperature estimates as well.

The final section states and proves our principal result (Theorem 4) on the hightemperature behaviour of Green functions in the renormalization scheme given. Since the main technical prerequisites are introduced in the preceding sections, the proof of the final theorem is comparatively easy. The extent to which this theorem actually leads to dimensional reduction is briefly alluded to, but is discussed exhaustively in another publication [17]. 


\section{BPHZ- and Decoupling Theorem United}

As a warmup-exercise we will prove the Euclidean BPHZ theorem as well as the Appelquist-Carazzone decoupling theorem [4, 2, 20,9] at one stroke. For simplicity we restrict ourselves to massive scalar theories without derivative coupling. The inclusion of spin would mainly present notational complications [28, 2], whereas the extension to the case where some of the masses vanish, while the external momenta remain off-shell and nonexceptional, presents some technical obstacles irrelevant for our purpose. In any case, these can be surmounted using results in $[30,20]$.

Initially our proof closely follows the already quite simple proof of the Minkowskian BPHZ theorem by Anikin et al. [3]. We then adjust and simplify their finale to arrive at a bound on the renormalized Feynman integral which is finite, and proportional to a negative power of the heavy mass, thus establishing the decoupling theorem. Since all estimates we use are trivial, our proof is technically considerably simpler than the demonstration of the Appelquist-Carazzone theorem given by Ambjørn [2].

Our starting point is the following expression for a generic $d$-dimensional ( $d$ even) Euclidean Feynman integral renormalized according to the BPHZ prescription:

$$
\begin{aligned}
F= & \int_{0}^{1}\left\{\prod_{i \in \Delta} \frac{d \zeta_{i}}{\delta_{i} !}\left(1-\zeta_{i}\right)^{\delta_{i}}\left(\frac{\partial}{\partial \zeta_{i}}\right)^{\delta_{l}+1}\right\} \int\left[\prod_{l=1}^{L} \frac{d^{d} k_{l}}{(2 \pi)^{d}} \frac{1}{k_{l}^{2}+m_{l}^{2}}\right] \\
& \cdot \prod_{v=1}^{V-C}(2 \pi)^{d} \delta^{(d)}\left(\zeta_{0} P_{v}-\prod_{l=1}^{L} \varepsilon_{v l} \tilde{\pi}_{l}(\zeta) k_{l}\right)
\end{aligned}
$$

This formula is equivalent to (2.2) below, which is given by [3], and can itself be derived from the formalism of Bergère and Zuber [6]. The integral $F$ corresponds to a Feynman diagram $\Gamma$ containing $L$ lines, $V$ vertices, $I$ loops, and $C$ connected components. $\Gamma$ contains superficially divergent subdiagrams $\left\{D_{i}\right\}_{i}$, labeled by an index $i$ in an index set $\Delta$, with degree of divergence $\delta_{i}=d I_{i}-2 L_{i}$. Neither $\Gamma$ nor the $D_{i}$ are necessarily connected [3], and $D_{0} \equiv \Gamma$. For the incidence matrix $\varepsilon$ to be well defined it is necessary to assume that $\Gamma$ contains no tadpole diagrams; this entails no loss of generality, for tadpoles vanish in the BPHZ scheme as well as in its generalization to finite temperature (cf. Sect. 4). The role of the structure involving $\zeta_{i}$ is to implement the subtractions at zero external momentum of the subdiagram $D_{i}$, as required by the BPHZ prescription. The ultimate $\zeta_{i}$-integration produces the finite remainder of the Taylor expansion of $D_{i}$ around zero momentum according to the Schlömilch formula [23]. Hence $\tilde{\pi}_{l}(\zeta)=\prod_{i}^{\prime} \zeta_{i}$, where the product is over all $i$ for which $k_{l}$ is an external momentum of $D_{i}$. If $\Gamma$ has no divergences then the $\zeta$-structure is to be omitted. Finally, $P_{v}$ is the total external momentum at the vertex $v$.

Introducing Feynman parameters $[28,26]$ one finds the parametric representation of $F$ :

$$
F=\int_{0}^{1}\left\{\prod_{i \in \Delta} \frac{d \zeta_{i}}{\delta_{i} !}\left(1-\zeta_{i}\right)^{\delta_{i}}\left(\frac{\partial}{\partial \zeta_{i}}\right)^{\delta_{i}+1} \zeta_{i}^{d I_{i}}\right\} \int_{0}^{\infty}\left(\prod_{l=1}^{L} d \alpha_{l} e^{-\alpha_{l} m_{l}^{2}}\right) e^{-E(\beta, P)} U(\beta)^{-\frac{1}{2} d}
$$


Here $\beta_{l}=\alpha_{l}\left(\pi_{l}(\zeta)\right)^{2}$, where $\pi_{l}(\zeta)=\prod_{i}^{\prime \prime} \zeta_{i}$, in which the product is over those $i \in \Delta$ for which $l \in D_{i}$. The $\zeta$-derivatives are understood to act on $\zeta$ as well as on $\beta$. In the derivation of (2.2) one encounters the matrix $d(\beta)_{v w}=\prod_{l=1}^{L} \varepsilon_{v l} \varepsilon_{w l} / \beta_{l}$, in terms of
which

$$
U(\beta)=\operatorname{det} d(\beta) \prod_{l=1}^{L} \beta_{l}=\sum_{T} \prod_{l \notin T} \beta_{l} .
$$

The sum is over all 1 -trees $T$ in $\Gamma$, a 1 -tree being a set of $V-C=L-I$ lines connecting all vertices in $\Gamma$ without including a loop ${ }^{1}$. Conversely, any set of $L-I$ lines not including a loop connects all vertices and must be a 1 -tree. A closed expression for $E(\beta, P)$ is known as well $[26,28]$, but here we only need the fact that it is analytic, non-negative, homogeneous of degree one in the $\beta_{l}$, and quadratic in the set of external momenta $P$.

We now pass from $\alpha_{l}$ to $\beta_{l}$ as integration variables (with Jacobian $\prod_{i} \zeta_{i}^{-2 L_{i}}$ ), and then decompose the $\beta$-integration region into $L$ ! sectors [15], in each of which $\beta_{i_{1}} \leqq \beta_{i_{2}} \leqq \ldots \leqq \beta_{i_{L}}$. For notational simplicity, take the sector $\left(i_{1}, \ldots, i_{L}\right)=(1, \ldots, L)$. We introduce the Speer variables [29]

$$
\beta_{l}=t_{l} t_{l+1} \ldots t_{L} ; \quad t_{l}=\beta_{l} / \beta_{l+1}
$$

with Jacobian $|\partial \beta / \partial t|=\prod_{l=1}^{L} t_{l}^{l-1}$. In each sector one has $t_{l} \in[0,1]$ for $l \neq L$, whereas $t_{L} \in[0, \infty[$. It follows from $(2.3)$ that $[28,29]$

$$
U(\beta)=\left(\prod_{l=1}^{L} t_{l}^{I_{l}}\right) u(t)
$$

where $u(t)$ is independent of $t_{L}$, and has the form $u(t)=1+f(t)$, with $f(t)$ analytic. $I_{L}$ is the number of loops in the diagram $\Gamma_{l}$ composed of the lines $1, \ldots, l$ (in an arbitrary sector this is to be replaced by $i_{1}, \ldots, i_{l}$ ). The integral (2.2) decomposes as a sum of integrals $F_{S}$ over a given sector.

As explained in more detail in [3] and [2], in the sector defined above, (2.2) can be written as

$$
\begin{aligned}
F_{S}= & \int_{0}^{1}\left\{\prod_{i \in \Delta} \frac{d \zeta_{i}}{\delta_{i} !}\left(1-\zeta_{i}\right)^{\delta_{i}} \zeta_{i}^{-1}\right\} \int_{0}^{\infty} d t_{L} \int_{0}^{1}\left(\prod_{l=1}^{L-1} d t_{l}\right)\left(\prod_{l=1}^{L} t_{l}^{l-1} e^{-\alpha_{l}(t, \zeta) m_{l}^{2}}\right) \\
& \cdot\left(\prod_{i \in \Delta} \mathscr{L}_{i}\right) e^{-t_{L} \tilde{E}(t, P)} u(t)^{-\frac{1}{2} d} \prod_{l=1}^{L} t_{l}^{-\frac{1}{2} d I_{l}} .
\end{aligned}
$$

Here $\widetilde{E}$ is independent of $t_{L}$, which has been explicitly factorized. The differential

\footnotetext{
${ }^{1}$ This generalizes the standard definition for a connected graph [26]. The expression given in [3] [their Eq. (7)] is not correct for disconnected graphs. Our generalized form will appear in Sect. 4
} 
operators $\mathscr{L}_{i}$ act on everything to their right, and are defined by

$$
\mathscr{L}_{i}=\prod_{s=0}^{\delta_{i}}\left[d I_{i}-s+2 \sum_{l \in D_{i}}\left(t_{l} \frac{\partial}{\partial t_{l}}-t_{l-1} \frac{\partial}{\partial t_{l-1}}\right)\right] .
$$

We define $\delta_{l}$ as the degree of divergence of $\Gamma_{l}$. For a given $l$ there are two possibilities:

1. $\delta_{l}<0$; the structure of (2.6) and (2.7) then implies that the integrand of (2.6) depends on $t_{l}$ as $t_{l}^{-1-\frac{1}{2} \delta_{l}}$ times an analytic function (note that $\delta_{l}$ must be even in even-dimensional scalar theories; in the general case an extra factor $t_{l}^{1 / 2}$ could occur).

2. $\delta_{l} \geqq 0$; then $\Gamma_{l}=D_{i}$ for some $i \in \Delta$, in which case the divergent subdiagram $D_{i}$ is called subordinate to the given sector [3]. Then, however, $\mathscr{L}_{i}$ assumes the simple form

$$
\mathscr{L}_{i}^{\text {sub }}=\prod_{s=0}^{\delta_{l}}\left[s+2 l+t_{l} \frac{\partial}{\partial t_{l}}\right] .
$$

Each term in (2.8) annihilates the corresponding term $t_{l}^{-l-\frac{1}{2} s}$ in the Laurent expansion of a function $f\left(t_{l}^{1 / 2}\right)$. If $f=f\left(t_{l}\right)$, as in (2.6), then the most singular term that survives is $t_{l}^{1-l}$, which combines with the $t_{l}^{l-1}$ already present in (2.6), so that the remainder is analytic in $t_{l}$. (If $d$ is odd, or if there is spin, then one could have extra square roots, which lead to the replacement of 1 by $\frac{1}{2}$ in the max-function in (2.9) below.) If $D_{0}=\Gamma$ is divergent, then $\mathscr{L}_{0}=\mathscr{L}_{0}^{\text {sub }}$ by acting on $e^{-t_{L} \tilde{E}} t_{L}^{-\frac{1}{2} d I}$ generates a factor $\widetilde{E}^{1+\frac{1}{2} \delta 0}$. More factors of $\widetilde{E}$ and its derivatives $\widetilde{E}^{(n)}$ are generated by (2.8) for $i \neq 0$, but these extra factors all occur in the combination $\left(t_{L} \widetilde{E}^{(n)}\right)^{m} e^{-t_{L} \widetilde{E}}$ and thence are irrelevant (see below).

Collecting these remarks, $F_{S}$ is found to have the structure

$$
\begin{aligned}
F_{S}= & \int_{0}^{1}\left\{\prod_{i \in \Delta} \frac{d \zeta_{i}}{\delta_{i} !}\left(1-\zeta_{i}\right)^{\delta_{i}} \zeta_{i}^{-1}\right\} \int_{0}^{\infty} d t_{L} \int_{0}^{1}\left(\prod_{l=1}^{L-1} d t_{l}\right) \\
& \cdot\left[\prod_{l=1}^{L} e^{-\alpha_{l}(t, \zeta) m_{l}^{2}} t_{l}^{-1+\max \left\{1,-\frac{1}{2} \delta_{l}\right\}}\right] w(t, P),
\end{aligned}
$$

where $w$ is analytic in each $t_{l}$, exponentially bounded in $t_{L}$, and of order $\varrho=2 \max \left\{0,1+\frac{1}{2} \delta_{0}\right\}$ in $P$ for $t_{L} \rightarrow 0$. Using the explicit form of $\widetilde{E}[28,26]$ and the compactness of the $t_{l}(l \neq L)$ integration region, we may estimate $\widetilde{E}(t, P) \leqq c_{1}(P)$, with $c_{1}$ quadratic in $P$. Also $t_{L}^{m} e^{-t_{L} x} \leqq\left(e x^{m}\right)^{-1}$, so that $\left(t_{L} \widetilde{E}^{(n)}\right)^{m} e^{-t_{L} \tilde{E}} \leqq c_{2}^{(n)}(P)$ which is of order $\varrho$ in $P$. Furthermore, $t_{l} \leqq 1$ trivially for $l \neq L$.

We now transform back to the $\beta_{l}$-variables, with Jacobian

$$
|\partial t / \partial \beta|=\prod_{l=2}^{L} \beta_{l}^{-1} \leqq \prod_{l=1}^{L} \beta_{l}^{-1+1 / L},
$$

as $\beta_{1} \leqq \beta_{2} \leqq \ldots \leqq \beta_{L}$ in the sector under study. After this estimate has been made, we extend the $\beta_{l}$-integration domain from $\beta_{l+1}$ to infinity (giving a further bound by positivity of the integrand). We then pass from $\beta_{l}$ to $\alpha_{l}$ as integration variables, and 


$$
\begin{aligned}
& \text { use } \prod_{l=1}^{L} \pi_{l}(\zeta)=\prod_{i \in \Delta} \zeta_{i}^{L_{i}} \text {. This gives the estimate }\left(\delta_{L}=\delta_{0}\right) \\
& \qquad \begin{array}{c}
F_{S} \leqq c(P) \int_{0}^{1}\left(\prod_{i} d \zeta_{i} \zeta_{i}^{-1+2 L_{i} / L}\right) \int_{0}^{\infty} d \alpha_{L} \alpha_{L}^{\max \left\{1,-\frac{1}{2} \delta 0\right\}-2+1 / L} \\
\cdot e^{-\alpha_{L} m_{L}^{2}} \int_{0}^{\infty} \prod_{l=1}^{L-1} d \alpha_{l} \alpha_{l}^{-1+1 / L} e^{-\alpha_{l} m_{l}{ }^{2}} \\
=K c(P) m_{L}^{2-2 \max \left\{1,-\frac{1}{2} \delta 0\right\}-2 / L} \prod_{l=1}^{L-1} m_{l}^{-2 / L}
\end{array}
\end{aligned}
$$

where $K$ is a finite constant which is trivially calculable from (2.10).

Let us divide the set of masses $\left\{m_{l}\right\}_{l}$ into two disjoint subsets $\left\{\mu_{l}\right\}_{l}$ and $\left\{M_{l}\right\}_{l}$, of light and heavy masses, respectively. The latter is supposed to be nonempty. We write (symbolically) $F=F\left(P^{2}, \mu^{2}, M^{2}\right)$, and scale the heavy masses by $\lambda^{\frac{1}{2}}$. Dimensionality then gives $F\left(P^{2}, \mu^{2}, \lambda M^{2}\right)=\lambda^{\frac{1}{2} \delta 0} F\left(P^{2} / \lambda, \mu^{2} / \lambda, M^{2}\right)$. Recalling that $c\left(P^{2} / \lambda\right)$ $=\lambda^{-\varrho / 2} c\left(P^{2}\right)$, it follows from (2.10) and the above that

$$
F_{S}\left(P^{2}, \mu^{2}, \lambda M^{2}\right) \leqq f \lambda^{\frac{1}{2} \delta_{0}-\max \left\{0,1+\frac{1}{2} \delta_{0}\right\}+\max \left\{1,-\frac{1}{2} \delta_{0}\right\}-1+N / L}
$$

with $f$ independent of $\lambda$, and $N$ the total number of light masses. Since at least one mass is heavy, one has $N<L$, so that (2.11) leads to the estimate $F_{S}(\lambda) \leqq f \lambda^{-\varepsilon}$, with $\varepsilon=1-N / L>0$.

This bound holds in each sector $\beta_{i_{1}} \leqq \beta_{i_{2}} \leqq \ldots \leqq \beta_{i_{L}}$, as the proof above can be trivially modified by permuting the indices $l=1, \ldots, L$. Since $F=\sum_{S} F_{S}$, which is a sum over $L$ ! sectors, we have proved the following decoupling theorem:

Theorem 1. Let $F\left(P^{2}, \mu^{2}, M^{2}\right)$ be a Feynman amplitude renormalized according to the $B P H Z$ subtraction scheme, depending on a set of external momenta $\{P\}$, a set of heavy masses $\left\{M_{l}\right\}$ (collectively denoted by $M$ ), and possibly a set of light masses $\left\{\mu_{l}\right\}$. For large $\lambda, F$ satisfies the bound

$$
F\left(P^{2}, \mu^{2}, \lambda M^{2}\right) \leqq \lambda^{-\varepsilon} f\left(P^{2}, \mu^{2}, M^{2}\right)
$$

for some $\varepsilon>0$ and some finite function $f$.

This proves the BPHZ theorem, which merely states that $F$ is finite, as well.

\section{Leading Large-Mass Behaviour}

To improve the bound (2.12), we wish to determine the leading term in the asymptotic expansion of $F$ in $1 / M$. Following the paradigm set by Bergère et al. in their study of the large- $P$ behaviour of Feynman amplitudes $[7,8]$ we take the Mellin-transform of $F\left(P^{2}, \mu^{2}, \lambda M^{2}\right)$ with respect to $\lambda$, and deduce the asymptotic expansion coefficients from the analytic structure of the transformed function.

For the Mellin transform to be of any use in this context we need the existence of $\lim _{\lambda \rightarrow 0} F\left(P^{2}, \mu^{2}, \lambda M^{2}\right)$. This limit actually exists if the external momenta are non$\lambda \rightarrow 0$ exceptional and off-shell $[30,20]$, so we will assume that this condition is satisfied. 
For $\mu \neq 0$ the final result may then trivially be continued to any value of the external momenta. Thus we define the Mellin transform

$$
\Phi(z)=\int_{0}^{\infty} d \lambda \lambda^{z-1} F(\lambda)
$$

where the dependence on $P^{2}, \mu^{2}$, and $M^{2}$ is suppressed. By the usual theory [10] and the bound (2.12) in combination with the existence of $F(0)$, it follows that $\Phi(z)$ is analytic at least in the strip $0<\Re z<\varepsilon$. We then have the inversion formula [10]

$$
F(\lambda)=\int_{c-i \infty}^{c+i \infty} \frac{d z}{2 \pi i} \lambda^{-z} \Phi(z)
$$

where $0<c<\varepsilon$ for some real $c$. An asymptotic expansion of $F$ in $\lambda^{-1}$ can now be obtained by shifting the contour to the right, and picking up the poles [11]. A pole $\Phi(z) \sim c_{p q}(z-p)^{-q}$ for $z \rightarrow p$ then obviously corresponds to a term $(-1)^{q} c_{p q}((q-1) !)^{-1} \lambda^{-p}(\log \lambda)^{q-1}$ in $F(\lambda)$, cf. [7].

We write $F$ as a sum over sectors, and study the asymptotic expansion of $F_{S}(\lambda)$ in the sector $\beta_{1} \leqq \beta_{2} \leqq \ldots \leqq \beta_{L}$, the generalization to arbitrary sectors being a trivial notational matter. We denote the set of "heavy" lines by $H$ (i.e. $l \in H$ if $m_{l}$ is heavy) and the set of "light" lines by $S$ (for "small"). We now scale each heavy mass in (2.9) according to $m_{l}^{2} \rightarrow \lambda m_{l}^{2}$ for $l \in H$, and take the Mellin transform with respect to $\lambda$. Interchanging the $\lambda$ - and $t$-integration order is allowed by absolute convergence, and the result is

with

$$
\Phi_{S}(z)=\int_{0}^{\infty} d t_{L} \int_{0}^{1}\left(\prod_{l=\lambda}^{L-1} d t_{l}\right)\left(\prod_{l=\lambda}^{L} t_{l}^{-z+\max \left\{1,-\frac{1}{2} \delta_{l}\right\}-1}\right) h\left(t_{\lambda}, \ldots, t_{L}, z\right)
$$

$$
\begin{aligned}
h\left(t_{\lambda}, \ldots, t_{L}, z\right)= & \int_{0}^{1}\left(\prod_{l=1}^{\lambda-1} d t_{l}\right) w(t, P) \int_{0}^{1}\left\{\prod_{i \in \Delta} \frac{d \zeta_{i}}{\delta_{i} !}\left(1-\zeta_{i}\right)^{\delta_{l} \zeta_{i}^{-1}}\right\} \\
& \cdot \Gamma(z)\left[\sum_{l \in H} M_{l}^{2} \frac{\beta_{l}}{\beta_{\lambda} \pi_{l}^{2}}\right]^{-z} e^{-\sum_{l \in S} \mu_{l}^{2} \beta_{l} / \pi_{l}^{2}} .
\end{aligned}
$$

We have defined $\lambda$ to be the largest element of $H$ in the given sector (that is, $\beta_{l} \leqq \beta_{\lambda}$ for all $l \in H$ ), while $\beta_{l}$ is defined by (2.4) and $\pi_{l}(\zeta)$ is given below (2.2). The structure of $h$ is determined by the following

Lemma 1. The function $h$ defined in (3.4) is:

1. analytic in $z$ for $\mathfrak{R} z>0$;

2. $\mathrm{C}^{\infty}$ in each $t_{l}>0$, and exponentially bounded for $t_{L} \rightarrow \infty$;

3. given by $h=\prod_{l=\lambda}^{L}\left(\log t_{l}\right)^{n_{l}} f\left(t_{\lambda}, \ldots, t_{L}, z\right)$ for $t_{l} \rightarrow 0$, where $f$ is $C^{\infty}$, and the positive integer $n_{l}$ is smaller than or equal to the total number of divergent diagrams containing the line $l$.

Proof. By (2.12), $h$ is analytic for $0<\mathfrak{R} z<\varepsilon$. In this region we write $\Gamma(z) x^{-z}$ $=\int_{0}^{\infty} d \varrho \varrho^{z-1} e^{-\varrho x}$, where $x$ is the expression between square brackets in (3.4). We 
also write $e^{-x_{l}}=\int_{c-i \infty}^{c+i \infty} \frac{d z}{2 \pi i} x_{l}^{-z_{l}} \Gamma\left(z_{l}\right)$ for $0<c<1 / L$, where $x_{l}$ is any suitable quantity appearing in (3.4) after the first-mentioned substitution has been made. Using $\prod_{l=1}^{L} \pi_{l}^{-z_{l}}=\prod_{i \in \Delta} \zeta_{\mathrm{i}}^{-} \sum_{l \in D_{i}} z_{l}$, and estimating $1-\zeta_{i} \leqq 1$, the $\zeta$-integrations can be done to give (omitting a few trivial constants)

$$
\begin{gathered}
h \leqq \int_{0}^{1}\left(\prod_{l=1}^{\lambda-1} d t_{l}\right) w(t, P) \int_{0}^{\infty} d \varrho \varrho^{z-1} \int_{c-i \infty}^{c+i \infty}\left(\prod_{l=1}^{L} \frac{d z_{l}}{2 \pi i} \Gamma\left(z_{l}\right)\right) \\
. \frac{\left(\prod_{l=1}^{L}\left(\mu_{l}^{2} \beta_{l}\right)^{-z_{l}}\right)\left(\prod_{l \in H}\left[M_{l}^{2} \varrho \beta_{l} / \beta_{\lambda}\right]^{-z_{l}}\right)}{\prod_{i \in \Delta} \sum_{l \in D_{l}} z_{l}} .
\end{gathered}
$$

The small- $t$ behaviour of the integrand may be inferred by moving the $z_{l}$-contours consecutively to the left, picking up the residue of the pole at $z_{l}=0$. The claims 2 and 3 then easily follow. The exponential decay in $t_{L}$ follows from the structure of $w(t, P)$, cf. Sect. 2. Analyticity in $z$ for $\mathfrak{R} z>1 / L$ is immediate from the above representation: the $z_{l}$-integrands have no singularity in the right-hand halfplane, so that according to a standard theorem in asymptotic theory [11] the $\varrho$-integrand decreases faster than any power for $\varrho \rightarrow \infty$. The analyticity in the strip $0<\mathfrak{R} \leqq 1 / L$ is a consequence of the analyticity of $\Phi_{S}(z)$ [cf. (3.3)] in this strip, and follows from the existence of the zero-mass limit, as explained before.

This lemma allows us to use the well-known [13] (and quite trivial) theorem stating that the function, defined for $\mathfrak{R} w>0$ by

$$
f(w)=\int_{0}^{\infty} d t t^{w}(\log t)^{n} g(t)
$$

for $n \in \mathbf{N}$ and $g(t) \in C^{\infty}\left(\mathbf{R}^{+}\right)$, has a meromorphic continuation to the entire complex plane, with simple poles in $w=-1,-2,-3, \ldots$ Proceeding as described below (3.2), it thus follows that the first pole of $\Phi_{S}(z)$ in (3.3) is encountered in

$$
z=\min _{l \in\{\lambda, \ldots, L\}} \max \left\{1,-\frac{1}{2} \delta_{l}\right\}
$$

The minimum is determined among the graphs $\Gamma_{\lambda}, \Gamma_{\lambda+1}, \ldots, \Gamma_{L}$ which, by definition of $\lambda$, each contain all heavy lines in $\Gamma$. The order of the pole is equal to the number of diagrams for which this minimum is simultaneously being assumed. Given the smallest line $l$ (in the sense of sectors) for which the minimum is assumed by $\Gamma_{l}$, other diagrams $\Gamma_{l^{\prime}}, l^{\prime}>l$, can possibly have the same value of $\max \left\{1,-\frac{1}{2} \delta_{l}\right\}$ only if one or more loops are added to $\Gamma_{l}$ (otherwise the degree of divergence obviously lowers by adding lines). A similar result holds in any sector. We now scale $M$ rather than $M^{2}$ by a factor $\lambda$. The remarks below (3.2), combined with the above information, then lead to 
Theorem 2. Let $F$ be as in Theorem 1. The leading term in the asymptotic expansion of $F\left(P^{2}, \mu^{2}, \lambda^{2} M^{2}\right)$ in $\lambda$ is equal to or smaller than a $\lambda$-independent factor times

$$
\lambda^{-\min _{\gamma} \max \left\{2,-\delta_{\gamma}\right\}}(\log \lambda)^{I},
$$

where the minimum is taken among those subdiagrams $\gamma \in \Gamma$ which contain all heavy lines. Here $\delta_{\gamma}$ is the superficial degree of divergence of $\gamma$, while I is the number of loops in $\Gamma$.

For theories in odd dimensions, and/or with spin, or derivative couplings, the bound is slightly worsened: 2 is replaced by 1 in the above result. In any case, we trivially have

Corollary 1. Let $F$ be a finite unrenormalized Feynman amplitude, otherwise as in Theorem 1. Then the leading term in the sense of Theorem 2 is

$$
\lambda^{\max _{\gamma} \delta_{\gamma}}(\log \lambda)^{I}
$$

where the maximum is determined among the subdiagrams $\gamma \in \Gamma$ containing all heavy lines.

It should be remarked that factors of $M$ should be included in the determination of the superficial degree of divergence (i.e. $k_{\mu} k_{v} / M^{2}$ has degree zero). Also, the leading terms may cancel out, so that the power of $\lambda$ given is an upper bound, as is the power of $\log \lambda$.

The corollary is equivalent to a theorem of Caswell and Kennedy [9], who state that the maximum is to be determined among diagrams which are a union of disjoint light-particle irreducible subgraphs [9] which contain all of the heavy-particle lines. To see the equivalence with our result, it is sufficient to remark that the degree of divergence of the light-particle reducible graphs containing all heavy lines can always be increased by deleting light particle lines.

The more general Theorem 2 is contained in the work of Manoukian [20], who, like Caswell and Kennedy, relied on momentum-space considerations. To the best of our knowledge, this section contains the first proof of a large-mass bound in the parametric formalism.

For pedagogical reasons we will now rederive Theorem 2 using the Kontorovich-Lebedev transform rather than the Mellin transform. This is a very useful exercise preparing for the high-temperature case in which, as we shall see, the latter is of no use. Besides, this derivation is independent of the preliminary bound (2.12), equivalent to the decoupling theorem, which is itself a consequence of Theorem 2.

Actually we will use a generalization of the Kontorovich-Lebedev transform given by Davies [10]. The transform pair reads

$$
\begin{gathered}
F(\lambda)=\int_{-i \infty}^{i \infty} \frac{d v}{\pi i} v I_{v}(k \lambda) \mathscr{F}(k, v) ; \\
\mathscr{F}(k, v)=\int_{0}^{\infty} \frac{d \lambda}{\lambda} K_{v}(k \lambda) F(\lambda) .
\end{gathered}
$$


Here $k>0$ is real, and $I_{v}$ and $K_{v}$ are modified Bessel functions. Using the asymptotic forms [19]

$$
I_{v}(x) \rightarrow \frac{x^{v}}{2^{v} v !} ; \quad K_{v}(x) \rightarrow 2^{v-1} \Gamma(v) x^{-v}
$$

for $x \rightarrow 0$, we see that the limit $k \rightarrow 0$ may be taken in (3.5), (3.6), in which case the transform reduces to the Mellin transform with argument $-z$. According to Davies [10], the generalized transform pair is valid at the points of continuity of $F(\lambda)$ if it satisfies Dirichlet's conditions, and if $F=\mathcal{O}(1)$ for $\lambda \rightarrow 0$ and $o\left(e^{\lambda}\right)$ for $\lambda \rightarrow \infty$ (note that $K_{v}(x) \rightarrow\left(\frac{\pi}{2 x}\right)^{\frac{1}{2}} e^{-x}$ for $\left.x \rightarrow \infty\right)$.

The function $F_{S}^{\alpha}(\lambda)=\lambda^{\alpha} F_{S}(\lambda)$, where $F_{S}(\lambda)$ is obtained by scaling the heavy masses $m_{l}^{2} \rightarrow \lambda m_{l}^{2}$ in (2.9), and $\alpha>0$ may be chosen at our convenience, obviously satisfies these conditions. In order to have a convenient representation of $\mathscr{F}_{S}^{\alpha}(\lambda)$ we perform a calculation

$$
\begin{aligned}
\int_{0}^{\infty} d \lambda \lambda^{\alpha-1} e^{-a \lambda} K_{v}(k \lambda) & =a^{-\alpha} \int_{0}^{\infty} \frac{d \lambda}{\lambda} f\left(\frac{k}{a \lambda}\right) K_{v}(\lambda) \\
& =\frac{1}{4} \int_{c-i \infty}^{c+i \infty} \frac{d z}{2 \pi i}\left(\frac{k}{2}\right)^{-z} a^{z-\alpha} \Gamma(\alpha-z) \Gamma\left(\frac{z+v}{2}\right) \Gamma\left(\frac{z-v}{2}\right),
\end{aligned}
$$

with $|\mathfrak{R} v|=0<c<\alpha$. Here $f(x)=x^{-\alpha} e^{-1 / x}$. The second equality follows, because the second member of (3.8) is precisely the Mellin-type convolution of $K_{v}$ with $f$ [24], with $k / a$ as the free variable. The Mellin-Barnes representation then follows simply by multiplying the Mellin transforms of $K_{v}$ and $f$. This also gives the condition on $c$ [24]. Using (3.8) with $a=\sum_{l \in H} \alpha_{l} m_{l}^{2}$ together with (3.5), (3.6) then yields the representation

$$
\begin{aligned}
F_{S}^{\alpha}(\lambda)= & \int_{-i \infty}^{i \infty} \frac{d v}{4 \pi i} v I_{v}(k \lambda) \int_{c-i \infty}^{c+i \infty} \frac{d z}{2 \pi i}\left(\frac{k}{2}\right)^{-z} \Gamma\left(\frac{z+v}{2}\right) \Gamma\left(\frac{z-v}{2}\right) \\
& \cdot \int_{0}^{\infty} d t_{L} \int_{0}^{1}\left(\prod_{l=\lambda}^{L-1} d t_{l}\right)\left(\prod_{l=\lambda}^{L} t_{l}^{z-\alpha+\max \left\{1,-\frac{1}{2} \delta_{l}\right\}-1}\right) h\left(t_{\lambda}, \ldots, t_{L}, \alpha-z\right),
\end{aligned}
$$

with $h$ given by (3.4), and $\lambda$ defined prior to (3.3).

The essence of our method is to take the limit $k \rightarrow 0$ in a controlled way. To do so, we interchange the $v$ - and $z$-integrations (allowed, as $|\Gamma(x+i y)| \rightarrow e^{-\frac{1}{2} \pi|y|}|y|^{x-\frac{1}{2}}$ for $y \rightarrow \infty$ ), and move the $v$-contour to the right, picking up the pole in $v=z$. We then let $k \rightarrow 0$, using (3.7); the shifted $v$-integral then vanishes because $\mathfrak{R} l \leqq \mathfrak{R} z$. The net result is

$$
F_{S}^{\alpha}(\lambda)=\int_{c-i \infty}^{c+i \infty} \frac{d z}{2 \pi i} \lambda^{z} \Phi_{S}(\alpha-z),
$$

cf. (3.2) and (3.3). Using the analyticity properties of $\Phi_{S}$ found in Lemma 1 and (3.3), we can move the contour to the left, and pass $\mathfrak{R} z=0$. We then let $\alpha \rightarrow 0$, after which we are back to (3.3), and can complete the proof of Theorem 2 accordingly. 
As promised, the bound (2.12) has not been employed: the relevant analyticity properties followed from a direct inspection of the integrands. We shall see in Sect. 5 that the above method can be used in a situation where the Mellin transform cannot be used at all. Since on the other hand the Mellin transform is a special case of the generalized Kontorovich-Lebedev transform, the latter is a more powerful, yet more complicated technique in asymptotic analysis than the former.

\section{Temperature-Dependent BPHZ Renormalization}

The aim of the remainder of this paper is to probe the infinite-temperature limit of a renormalizable relativistic thermal field theory. We will use the conventional imaginary-time (Matsubara) formulation [18], in which the bosonic fields are defined on a Euclidean time interval which is a circle with circumference $\beta=1 / T$. Fermionic fields are functions on the same compact interval, but rather satisfy antiperiodic boundary conditions [18]. In momentum-space perturbation theory one must therefore replace the continuous (Euclidean) energy $k_{4}$ by a discrete frequency $\omega_{n}=2 \pi T n$ (bosons) or $2 \pi T\left(n+\frac{1}{2}\right)$ (fermions), where $n \in \mathbf{Z}$ must be summed over in loops. We will refer to the $\omega_{n}=0$ terms as static modes, all the other modes being nonstatic. For large temperatures the nonstatic modes obviously behave like fields with a very large mass, suggesting their decoupling in analogy with the AppelquistCarazzone theorem [5]. In the following we restrict ourselves to scalar fields for simplicity.

Consider a diagram $\Gamma$, and define $L, V, I$, and $C$ as in Sect. 2. Since we eventually wish to find out whether static diagrams can be approximated by omitting nonstatic modes in internal loops, we assume that all external frequencies to $\Gamma$ vanish. This is just to simplify the expressions; all proofs below easily extend to the general case. Let now $M$ be a partial finite-temperature Feynman (-Matsubara) amplitude corresponding to $\Gamma$. By "partial" we here mean that a given number of $I^{N}$ frequency summations are carried out (omitting the zero mode), whereas in the other $I^{S}=I-I^{N}$ loops only the zero mode is taken into account, the sum over the other modes being deleted. The total amplitude is obviously the sum over all possible partial amplitudes. Corresponding to a given choice of energy routing in $\Gamma$, there is a set of lines $S$ (which may be empty, especially if the external lines are nonstatic) whose frequency $\omega_{n}$ vanishes whatever the choice of the frequencies in the nonstatic loops. We now define $N$ to be the diagram $\Gamma-S$, i.e. the subdiagram of $\Gamma$ obtained by deleting the lines in the set $S$ (isolated vertices thus emerging are to be ignored, of course). The topology of $N$ is independent of the energy routing chosen in $\Gamma$.

In principle, the finite temperature theory can be renormalized by subtractions at $T=0$ [18]. Zero-temperature renormalization prescriptions do not lead to decoupling of the nonstatic modes at high temperature, though [17] (in the same sense that mass-independent renormalization prescriptions at zero temperature, like minimal subtraction, do not lead to the decoupling of heavy particles, i.e. violate the Appelquist-Carazzone theorem). Instead, we renormalize by means of zero-momentum subtractions at temperature $T$. The superficial degree of divergence of a diagram $\Gamma_{i}$ containing $I_{i}^{S}$ static loops is given by

$$
\tau_{i}=\delta_{i}-I_{i}^{S}=(d-1) I_{i}+I_{i}^{N}-2 L_{i},
$$


where $I_{i}^{N}=I-I_{i}^{S}$ is the number of nonstatic loops in $\Gamma_{i}$. We denote the subdiagrams of $\Gamma$ (more precisely, of a given partial contribution to $\Gamma$ ) for which $\tau_{i} \geqq 0$ by $D_{i}, i \in \Delta$. These diagrams are renormalized at temperature $T$ by subtracting all terms in their Taylor expansion around zero spatial momentum up to order $\tau_{i}$. The external frequencies in the subtracted terms are put equal to zero (for fermions one still puts $n=0$, so that one subtracts at external frequencies $\omega=\pi T)$. For example, the global divergence in a bare quadratically divergent self-energy $\Sigma\left(\omega_{n}, \mathbf{p}, T\right)$ is renormalized according to

$$
\Sigma_{R}\left(\omega_{n}, \mathbf{p}, T\right)=\Sigma\left(\omega_{n}, \mathbf{p}, T\right)-\Sigma(0, \mathbf{0}, T)-\left(\mathbf{p}^{2} \frac{\partial}{\partial \mathbf{p}^{2}} \Sigma\right)(0, \mathbf{0}, T) .
$$

(It is also possible to subtract at the given value of $\omega_{n}$ rather than its zero value, at the expense of introducing $n$-dependent renormalized parameters. Theorem 4 in Sect. 5 can be shown to still hold in this more general scheme.)

As in the vacuum case [cf. (2.1)] we implement these subtractions by repeatedly using the Schlömilch formula for the remainder of a Taylor series. There is a tiny nuisance in the present case, because we subtract at a fixed $\omega_{n}=0$. The formula for the remainder of order $\tau+1$,

$$
\left(1-T_{\tau}\right) f(n, \mathbf{p})=\frac{1}{\tau !} \int_{0}^{1} d \zeta(1-\zeta)^{\tau}\left(\frac{\partial}{\partial \zeta}\right)^{\tau+1} f(\zeta n, \zeta \mathbf{p})
$$

can still be used, however, if we understand $\frac{\partial}{\partial \zeta}$ to act on $\zeta \mathbf{p}$ alone. In addition there is the seeming problem of assigning a meaning to $f(\zeta n)$, where $f(n)$ is defined for discrete integers only. Fortunately, formula (4.2), if interpreted as we did, effectively employs $f(\zeta n)$ in $\zeta=0$ and $\zeta=1$ only. Therefore, one may assign an arbitrary meaning to $f(\zeta n)$ as long as its values for $\zeta=0$ and $\zeta=1$ reduce to $f(0)$ and $f(n)$, respectively. In our application, $f(n)$ will be $\delta_{K}(n)=\delta_{n 0}$. We now interpret $\delta_{K}(\zeta n)$ as

$$
\delta_{K}(\zeta n)=\int_{0}^{1} d u e^{2 \pi i u \zeta n} .
$$

Thus we are led to the following partial amplitude, generalizing (2.1):

$$
\begin{aligned}
M= & T^{I} \int_{0}^{1}\left\{\prod_{i \in \Delta} \frac{d \zeta_{i}}{\tau_{i} !}\left(1-\zeta_{i}\right)^{\tau_{i}}\left(\frac{\partial}{\partial \zeta_{i}}\right)^{\tau_{i}+1}\right\} \\
& \cdot\left(\prod_{l \in N} \sum_{n_{l}=-\infty}^{\infty}\right) \int\left[\prod_{l=1}^{L} \frac{d^{d-1} k_{l}}{(2 \pi)^{d-1}} \frac{1}{k_{l}^{2}+\omega_{l}^{2}+m_{l}^{2}}\right] \\
& \cdot \prod_{v=1}^{V-C}(2 \pi)^{d-1} \delta^{(d-1)}\left(\zeta_{0} \mathbf{P}_{v}-\sum_{l=1}^{L} \varepsilon_{v l} \tilde{\pi}_{l}(\zeta) k_{l}\right) \delta_{K}\left(\sum_{l=1}^{L} \varepsilon_{v l} n_{l} \tilde{\pi}_{l}(\zeta)\right),
\end{aligned}
$$

where $\delta_{K}(m)=\delta_{m 0}$, and $\omega_{l}=2 \pi T n_{l}$ (note that $\tilde{\pi}_{l}(\zeta)$ is defined prior to (2.2)). The prime on the summation sign means that the zero mode is to be omitted. Since our renormalization scheme is rather hybrid, we cannot even appeal to heuristic results in finite temperature renormalization theory $[22,18]$. We therefore start with a convergence proof of (4.4). With more effort we could derive a bound à la (2.12) at 
the same stage, but we refrain from doing so, as we will give a much better bound in Theorem 4. In any case, many lemmata below will be essential in the proof of our final decoupling theorem as well.

Repeating, mutatis mutandis, the steps leading from (2.1) to (2.6), recalling that the $\zeta$-derivatives in (4.4) do not act on the Kronecker delta, we now obtain (in the sector $\beta_{1} \leqq \ldots \leqq \beta_{L}$, as always)

$$
\begin{aligned}
M_{S}= & T^{I} \int_{0}^{1}\left\{\prod_{i \in \Delta} \frac{d \zeta_{i}}{\tau_{i} !}\left(1-\zeta_{i}\right)^{\tau_{i}} \zeta_{i}^{-1-I_{i}^{N}}\right\} \int_{0}^{\infty} d t_{L} \int_{0}^{1}\left(\prod_{l=1}^{L-1} d t_{l}\right)\left(\prod_{l=1}^{L} e^{-\alpha_{l}(t, \zeta) m_{l}^{2}}\right) \\
& \cdot f(t) g(t, \zeta) .
\end{aligned}
$$

Here

$$
f(t)=\left(\prod_{l=1}^{L} t_{l}^{l-1}\right)\left(\prod_{i \in \Delta} \tilde{\mathscr{L}}_{i}\right) e^{-E(t, \mathbf{P})} u(t)^{-\frac{1}{2}(d-1)} \prod_{l=1}^{L} t_{l}^{-\frac{1}{2}(d-1) I_{l}},
$$

where $\tilde{\mathscr{L}}_{i}$ is given by making the replacements $\delta_{i} \rightarrow \tau_{i}$ and $d \rightarrow d-1$ in (2.7). Also

$$
g(t, \zeta)=\left(\prod_{l \in N} \sum_{n_{l}=-\infty}^{\infty} e^{-\alpha_{l}(t, \zeta) \omega_{l}^{2}}\right) \prod_{v} \delta_{K}\left(\sum_{l=1}^{L} \varepsilon_{v l} n_{l} \tilde{\pi}_{l}(\zeta)\right)
$$

with $\alpha_{l}=t_{l} \ldots \mathrm{t}_{L} /\left(\pi_{l}(\zeta)\right)^{2}$, and $\pi_{l}$ defined prior to (2.3). The final product is over those of the original $V-C$ vertices [cf. (4.4)] which are attached to the reduced diagram $N$. We firstly have

\section{Lemma 2.}

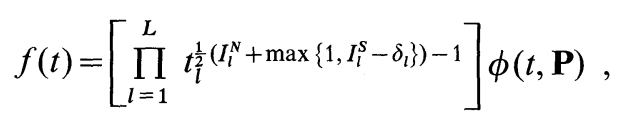

where $I_{l}^{N}$ and $I_{l}^{S}$ are the number of nonstatic and static loops in $\Gamma_{l}$, respectively. The function $\phi$ is exponentially bounded in $t_{L}$, and analytic in each $t_{l}$, modulo a possible factor of $t_{l}^{\frac{1}{2}}$ for each $t_{l}$, which occurs if $I_{l}^{N}$ is odd.

(Recall that $\Gamma_{l}$ is the diagram composed of the lines $1, \ldots, l$.) The proof of this lemma is entirely analogous to that of Eq. (2.9), so we omit it.

Lemma 2 above is a piece of cake compared to

\section{Lemma 3.}

$$
g(t, \zeta)=\left(\prod_{l=1}^{L} t_{l}^{-\frac{1}{2} I_{l}^{N}}\right)\left(\prod_{i \in \Delta} \zeta_{i}^{I_{i}^{N}}\right) \chi(t, \zeta),
$$

where $\chi$ is bounded and integrable in each variable $\left(I_{i}^{N}\right.$ is the number of nonstatic loops in $D_{i}$ ).

Proof. To save writing we set $T=1 / 2 \pi$ (or absorb $T$ into the $\alpha_{l}$ ). Recalling (4.3), we rewrite (4.7) as

$$
g=\int_{0}^{1}\left(\prod_{v} d u_{v}\right) \prod_{l \in N}\left[\vartheta\left(-\sum_{v} u_{v} \varepsilon_{v l} \tilde{\pi}_{l}(\zeta), i \alpha_{l} / \pi\right)-1\right],
$$

in terms of the Jacobi theta function (also denoted by $\vartheta_{00}$ or $\vartheta_{3}$ )

$$
\vartheta(z, \tau)=\sum_{n=-\infty}^{\infty} e^{i \pi \tau n^{2}+2 i \pi n z} .
$$


We then use the functional equation [25]

$$
\vartheta(z, \tau)=e^{-\pi i z^{2} / \tau} \vartheta(z / \tau,-1 / \tau) / \sqrt{-i \tau}
$$

and the definitions of $\pi_{l}(\zeta), \tilde{\pi}_{l}(\zeta), \beta_{l}$, and $d(\beta)$ [cf. text above (2.3)] to find

$$
\begin{aligned}
g= & \left(\prod_{i \in \Delta} \zeta_{i}^{I_{l}^{N}}\right)\left[\prod_{l \in N} \beta_{l} / \pi\right]^{-\frac{1}{2}}\left(\prod_{v} \int_{0}^{U_{v}} d u_{v}\right) e^{-\pi^{2} u_{v} d(\beta)_{v w} u_{w}} \\
& \cdot \prod_{l \in N}\left[\vartheta\left(i u_{v} \varepsilon_{v l} / \alpha_{l} \pi_{l}, i \pi / \alpha_{l}\right)-\sqrt{\alpha_{l} / \pi} e^{\pi^{2}\left(u_{v} \varepsilon_{v l}\right)^{2} / \alpha_{l}}\right]
\end{aligned}
$$

Here $U_{v}=\prod_{i}^{\prime} \zeta_{i}$, where the product is over all $i$ for which $v \in D_{i}$. It is understood that $v$ and $w$ are summed over if appropriate. Since $u_{v} \leqq U_{v}$, by definition of $\pi_{l}$ and $\varepsilon_{v l}$, we have $\left|u_{v} \varepsilon_{v l} \pi_{l}^{-1}\right| \leqq 1$ (no sum over $l$ ). The expression in square brackets is positive, so to obtain an upper bound on $g$ we may replace the last exponential by one. We then extend the $u$-integration ranges to the real axis, and use (2.3) and (4.11) to obtain (omitting some factors of $\pi$ )

and

$$
0<g<\left(\prod_{i \in \Delta} \zeta_{i}^{I_{i}^{N}}\right) U^{N}(\beta)^{-\frac{1}{2}} \prod_{l \in N}\left[h\left(\alpha_{l}\right)-\sqrt{\alpha_{l} / \pi}\right],
$$

$$
h\left(\alpha_{l}\right)=1+2 \sum_{n=1}^{\infty} e^{-\pi^{2} n^{2} / \alpha_{l}+2 \pi n / \alpha_{l}}
$$

Here $U^{N}$ is defined similar to (2.3); now, however, only lines in $N$ participate (that is, the one-trees $T$ must lie within $N$, whereas the lines $l \notin T$ must be in $N$ as well.

We will now analyze $U^{N}$. In the following we use the trivial fact that $I_{l}^{N}$ is both the number of nonstatic loops in $I_{l} \subset \Gamma$, and the number of loops in the graph $N_{l} \equiv I_{l} \cap N$. By definition of a 1-tree [cf. text below (2.3)], each term $\prod_{l \notin T} \beta_{l}$ contains $I_{\lambda}^{N}$ factors of the type $\beta_{l}$, where $l \in N_{\lambda}$; adding each of the corresponding lines to the given $T$ would form a loop. Therefore, by (2.4), $\prod_{l=1}^{L} t_{l}^{I_{l}^{N}}$ factorizes in $U^{N}$. Conversely, we can form a 1-tree in $N$ for which the last-mentioned product equals $\prod_{l \notin T} \beta_{l}$. This tree consists of the lines $l \in N$ for which $I_{l}^{N}=I_{l-1}^{N}\left(I_{-1} \equiv 0\right)$. This set obviously contains no loops, and has $L^{N}-I^{N}$ lines, so it must indeed be a 1-tree. Therefore, (2.5) generalizes to

$$
U^{N}(\beta)=\left(\prod_{l=1}^{L} t_{l}^{I_{l}^{N}}\right) u(t)
$$

where $u(t)$ satisfies the same conditions as in (2.5).

We proceed with the second factor in (4.14). We see immediately from (4.13) that it goes to one for $\alpha_{l} \rightarrow 0$ (small $t$ ), so that it remains to investigate its large- $\alpha_{l}($ small $\zeta$ ) behaviour. To do so, we use [27, Eq. I. (3.13)] to give a Mellin-Barnes representation of the sum [10]. We then insert the Mellin-Barnes integral representation [1, Eq. (19.5.13)] for the parabolic cylinder function ${ }^{2}$ and perform one of the two contour

\footnotetext{
${ }^{2}$ Correcting two typographical errors: $x \rightarrow z$ and "zeros" $\rightarrow$ "poles" in the text below (19.5.13)
} 
integrations. This yields

$$
h(\alpha)=1+\sum_{m=0}^{\infty} \frac{(\alpha / 4)^{-m / 2}}{m !} \int_{\sigma-i \infty}^{\sigma+i \infty} \frac{d z}{2 \pi i} \zeta(z) \Gamma\left(\frac{z+m}{2}\right)\left(\frac{\alpha}{\pi^{2}}\right)^{z / 2},
$$

with $\sigma>1$. An asymptotic expansion for $\alpha \rightarrow \infty$ then follows by shifting the contour to the left, and picking up the poles [11]. One finds that both the term 1 in (4.17) and the term $\sqrt{\alpha / \pi}$ in (4.14) are cancelled, and it follows that $h(\alpha)-\sqrt{\alpha / \pi}=\mathcal{O}(1)$ for $\alpha \rightarrow \infty$.

Combining this with (4.14) and (4.16) we thus have concluded the proof of Lemma 3.

Theorem 3. The renormalized partial Feynman-Matsubara amplitude defined by (4.4) is finite.

Proof. It is sufficient to prove that the contribution (4.5) from the sector $\beta_{1} \leqq \ldots \leqq \beta_{L}$ is finite: the other sectors differ by permutations of the index set $\{1, \ldots, L\}$, and the proof of the convergence of the analogues of (4.5) in these sectors consists of a trivial rewriting of the proof in the present sector.

By Lemma 3, the $\zeta$-integrand in (4.5) behaves like $\prod_{i \in \Delta} \zeta_{i}^{-1} \prod_{l=1}^{L} e^{-\alpha_{l} m_{l}^{2}}$ at worst for $\zeta_{i} \rightarrow 0$. The reasoning in the proof of Lemma 1 (Sect. 3) then shows that the $\zeta$-integration introduces logarithmic singularities in each $t_{l}$ at worst. Lemmata 2 and 3 together imply that the rest of each $t_{l}$-integrand is $\mathcal{O}\left(t_{l}^{-\frac{1}{2}}\right)$ for $t_{l} \rightarrow 0$, so that each $t_{l}$-integration is finite. The integrability for $t_{L} \rightarrow \infty$ follows from the exponential decay of the $t_{L}$-integrand. All interchanges of integration and summation orders are justified by the positivity of each integrand (apart from the innocent factor $\left.\left(1-\zeta_{i}\right)^{\tau_{i}} \leqq 1\right)$.

\section{High-Temperature Behaviour}

In this section we find a sharp bound on the leading term in the (asymptotic) hightemperature expansion of the renormalized Feynman-Matsubara integral (4.4). The main technical problem is that $M(T)=\mathcal{O}(1)$ for $T \rightarrow 0$ and $\mathcal{O}\left(T^{I-\gamma}\right)$ for $T \rightarrow \infty$, for some (as yet unknown) $\gamma>0$, which means that $M$ does not allow a Mellintransform with respect to $T$. Fortunately enough, the generalized KontorovichLebedev transform (3.5), (3.6) can be used.

We define $M^{\alpha}(\lambda)=\lambda^{\alpha} M(\lambda)$, where $M(\lambda)$ is obtained by scaling $\omega_{l}^{2} \rightarrow \lambda \omega_{l}^{2}$ in (4.4), i.e. by scaling $T^{2} \rightarrow \lambda T^{2}$ while ignoring the prefactor $T^{I}$ in (4.4) (it will be reinserted in Theorem 4 below). Using (3.8) we can give a representation of $M_{S}^{\alpha}(\lambda)$ [cf. (4.5)] similar to (3.9). For simplicity we set $T=1 / 2 \pi$, and find

$$
\begin{aligned}
M_{S}^{\alpha}(\lambda)= & \int_{-i \infty}^{i \infty} \frac{d v}{4 \pi i} v I_{v}(k \lambda) \int_{c-i \infty}^{c+i \infty} \frac{d z}{2 \pi i}\left(\frac{k}{2}\right)^{-z} \Gamma\left(\frac{z+v}{2}\right) \Gamma\left(\frac{z-v}{2}\right) \\
& \cdot \int_{0}^{\infty} d t_{L} \int_{0}^{1}\left(\prod_{l=\lambda}^{L-1} d t_{l}\right)\left(\prod_{l=\lambda}^{L} t_{l}^{z-\alpha+\frac{1}{2}\left(I_{l}^{N}+\max \left\{1, I_{l}^{S}-\delta_{l}\right\}\right)-1}\right) m\left(t_{\lambda}, \ldots, t_{L}, z\right),
\end{aligned}
$$


with

$$
\begin{aligned}
m= & \Gamma(\alpha-z) \int_{0}^{1}\left(\prod_{l=1}^{\lambda-1} d t_{l}\right) f(t) \int_{0}^{1}\left\{\prod_{i \in \Delta} \frac{d \zeta_{i}}{\tau_{i} !}\left(1-\zeta_{i}\right)^{\tau_{i}} \zeta_{i}^{-1-I_{l}^{N}}\right\} \prod_{l=1}^{L} e^{-\alpha_{l} m_{l}^{2}} \\
& \cdot\left(\prod_{l \in N} \sum_{n_{l}=-\infty}^{\infty}\right)\left[\sum_{l \in H} \frac{\beta_{l} n_{l}^{2}}{\beta_{\lambda}\left(\pi_{l}(\zeta)\right)^{2}}\right]^{z-\alpha} \prod_{v} \delta_{K}\left(\sum_{l=1}^{L} \varepsilon_{v l} n_{l} \tilde{\pi}_{l}(\zeta)\right) .
\end{aligned}
$$

Here $f(t)$ is given in (4.6), and $\beta_{l}$ by (2.4). In analogy to Sect. $3, \lambda$ is now defined as the largest line in $N$ in the given sector. We choose $\alpha=\frac{1}{2} I^{N}+\varepsilon$, for some $0<\varepsilon<\frac{1}{2}$. We recall from Sect. 3 that $c$ is originally constrained by $0<c<\alpha$, but to justify the interchange of summations and integrations used to derive (5.1) and (5.2) we here actually need $0<c<\varepsilon$. Also note that $I_{l}^{N}=I^{N}$ for $l \geqq \lambda$. The entire $t$-dependence of $m$ is in the factors $e^{-\alpha_{l} m_{l}^{2}}$ in (5.2). We have

Lemma 4. The function $m\left(t_{\lambda}, \ldots, t_{L}, z\right)$ defined by (5.2)

1. is analytic in $z$ for $\mathfrak{R} z<\varepsilon$;

2. satisfies conditions 2 and 3 of Lemma 1 (Sect. 3).

Proof. The frequency summation in (5.2) converges for $\beta_{l} \neq 0$ and $\mathfrak{R} z<\varepsilon$ by the theory of the Epstein $\zeta$-function [12]. To find its leading singilarity for $t_{l} \rightarrow 0$ we write $\Gamma(\alpha-z)(\ldots)^{z-\alpha}=\int_{0}^{\infty} d \varrho \varrho^{\alpha-z-1} e^{-\varrho(\ldots)}$, and put the $\varrho$-integration to the left of all the others (the integrand is positive and the integral exists a fortiori!). Since the prefactor of $n_{\lambda}^{2}$ never vanishes, the integrand decays exponentially for $\varrho \rightarrow \infty$. Following the proof of Lemma 3 , we find that the integrand is $\mathcal{O}\left(\varrho^{-\frac{1}{2} I^{N}}\right)$ for $\varrho \rightarrow 0$. In view of the choice $\alpha=\frac{1}{2} I^{N}+\varepsilon$, this proves point 1 provided that the other integrals are finite. These, however, are completely under control by Lemmata 2 and 3 in Sect. 4. Point 2 then follows as in the proof of Lemma 1.

We next eliminate the $v$-integration in (5.1) in the manner described prior to (3.10). This gives

$$
\begin{aligned}
M_{S}^{\alpha}(\lambda)= & \int_{c-i \infty}^{c+i \infty} \frac{d z}{2 \pi i} \lambda^{z} \int_{0}^{\infty} d t_{L} \int_{0}^{1}\left(\prod_{l=\lambda}^{L-1} d t_{l}\right) \\
& \cdot\left(\prod_{l=\lambda}^{L} t_{l}^{z-\alpha+\frac{1}{2}\left(I_{l}^{N}+\max \left\{1, I_{l}^{S}-\delta_{l}\right\}\right)-1}\right) m\left(t_{\lambda}, \ldots, t_{L}, z\right) .
\end{aligned}
$$

An asymptotic expansion for $\lambda \rightarrow \infty$ now follows by shifting the contour to the left and picking up the poles in complete analogy to the large-mass case (Sect. 3), where $z$ is replaced by $-z$. Lemma 4 guarantees that we may use the theorem quoted after the proof of Lemma 1 . We see that $\lambda^{\alpha}$ then factorizes in $M_{S}^{\alpha}(\lambda)$, as it should.

As usual, an expression of the type (5.3) is valid in any sector. Taking the factor $T^{I}$ in (4.2) into account, which was not scaled in the definition $M^{\alpha}(\lambda)$, and scaling $T$ rather than $T^{2}$ by a factor $\lambda$, we thus have arrived at the central result of this paper.

Theorem 4. Let $M(T, \mathbf{P}, m)$ be a partial Feynman-Matsubara amplitude corresponding to a diagram $\Gamma$ with $I$ loops, in which $I^{N}$ frequency summations are carried out, 
omitting the zero modes. Here $T$ is the temperature, and $M(T, \ldots)$ denotes the explicit $T$-dependence. Also, $\mathbf{P}$ stands for an arbitrary set of external momenta, and $m$ represents a set of nonvanishing masses on which $M$ depends. Let $M$ be renormalized by (marginal) zero-momentum subtractions at temperature T, as explained in Sect. 4. Then the leading term in the asymptotic expansion of $M(\lambda T, \mathbf{P}, m)$ for large $\lambda$ is bounded by

$$
c \lambda^{I-\min _{\gamma}\left(I_{\gamma}^{N}+\max \left\{1, I_{\gamma}^{S}-\delta_{\gamma}\right\}\right)}(\log \lambda)^{I},
$$

where $c$ is independent of $\lambda$. The minimum is determined among all subdiagrams $\gamma \subset \Gamma$ containing all nonstatic lines. $I_{\gamma}^{N}$ and $I_{\gamma}^{S}$ are the number of nonstatic and static loops in $\gamma$, respectively, and $\delta_{\gamma}$ is the superficial degree of divergence of $\gamma$ as determined by naive four-dimensional power counting.

The above bound is less complicated in the following case.

Corollary 2. Let $M(\lambda T, \mathbf{P}, m)$ be a finite unrenormalized partial Feynman-Matsubara amplitude, otherwise as in Theorem 4. Then the leading term in its asymptotic expansion for large $\lambda$ is bounded by

$$
c \lambda^{I+\max _{\gamma} \delta_{\gamma}^{(3)}}(\log \lambda)^{I},
$$

where $\delta_{\gamma}^{(3)}$ is the superficial degree of divergence of $\gamma$ as determined by threedimensional power counting. The maximum is determined among the diagrams $\gamma$ described in Theorem 4.

These results are valid for any spin and in any dimension, and include the case of nonzero external frequencies $\omega$. [These would show up in the Kronecker delta in (4.4) and (4.7), and in (4.10) in the guise of a factor $e^{i u_{v} \omega_{v}}$, not spoiling any proof.]

Since the purely static diagrams are naively proportional to $T^{I}$, and the nonstatic diagrams by Theorem 4 cause a suppression factor of $T^{-\frac{1}{2}}$ or more, it may appear that a dimensional reduction takes place in the infinite-temperature limit of thermal field theory, in the sense that the nonstatic modes decouple, and leave a three-dimensional theory behind. As discussed extensively in [17] there is a caveat, however: because the renormalization prescription in which Theorem 4 holds is temperature-dependent, the renormalized parameters, in particular the masses, are $T$-dependent. It can be shown [17] that dimensional reduction only occurs if $\operatorname{Lim} m(T) / T=0$, where the $T$-dependence of the masses can be evaluated by means $T \rightarrow \infty$

of the finite-temperature renormalization group [21, 17]. In those cases the results of this section constitute a rigorous proof that such a reduction mechanism indeed applies.

\section{References}

1. Abramowitz, M., Stegun, I.: Handbook of mathematical functions. New York: Dover 1965

2. Ambjørn, J.: On the decoupling of massive particles in field theory. Commun. Math. Phys. 67, 109-119 (1979)

3. Anikin, S.A., Zav'yalov, O.I., Polivanov, M.K. : A simple proof of the Bogolyubov-Parasyuk theorem. T.M.P. 17, 189-198 (1973), transl. ibid., 1082-1088 (1974) 
4. Appelquist, T., Carazzone, J.: Infrared singularities and massive fields. Phys. Rev. D11, 2856-2861 (1975)

5. Appelquist, T., Pisarski, R.D. : High-temperature Yang-Mills theories and three-dimensional quantum chromodynamics. Phys. Rev. D23, 2305-2317 (1981)

6. Bergère, M.C., Zuber, J.B.: Renormalization of Feynman amplitudes and parametric integral representation. Commun. Math. Phys. 35, 113-140 (1974)

7. Bergère, M.C., Lam, Y-M.P.: Asymptotic expansion of Feynman amplitudes. I. Commun. Math. Phys. 39, 1-32 (1974)

8. Bergère, M.C., de Calan, C., Malbouisson, A.P.C.: A theorem on asymptotic expansion of Feynman amplitudes. Commun. Math. Phys. 62, 137-158 (1978)

9. Caswell, W.E., Kennedy, A.D.: Asymptotic behavior of Feynman Integrals: Convergent integrals. Phys. Rev. D28, 3073-3089 (1983)

10. Davies, B.: Integral transforms and their applications. Berlin, Heidelberg, New York: Springer 1978

11. Dingle, R.B.: Asymptotic expansions: their derivation and interpretation. London, New York: Academic Press 1973

12. Erdélyi, A. et al.: Higher transcendental functions, Vol. III. New York, Toronto, London: McGraw-Hill Book 1955

13. Gelfand, I.M., Shilov, G.E.: Verallgemeinerte Funktionen (Distributionen), Bd. 1. Berlin: VEB Deutscher Verlag der Wissenschaften 1960

14. Gross, D.J., Pisarski, R.D., Yaffe, L.G.: QCD and instantons at finite temperature. Rev. Mod. Phys. 53, 43-80 (1981)

15. Hepp, K.: Proof of the Bogoliubov-Parasiuk theorem on renormalization. Commun. Math. Phys. 2, 301-326 (1966)

16. Jackiw, R.: Gauge theories in three dimensions (=at high temperature). In: Gauge theories of the eighties, pp. 157-219. Raitio, R., Lindfors, J. (eds.). SLNP 181. Berlin, Heidelberg, New York: Springer 1983

17. Landsman, N.P.: Limitations to dimensional reduction at high temperature. Nucl. Phys. B322, 498-530 (1989)

18. Landsman, N.P., van Weert, Ch.G.: Real- and imaginary time field theory at finite temperature and density. Phys. Rep. 145, 141-249 (1987)

19. Magnus, W., Oberhettinger, F., Soni, R.P.: Formulas and theorems for the special functions of mathematical physics, third ed. Berlin, Heidelberg, New York: Springer 1966

20. Manoukian, E.B.: Renormalization. New York, London: Academic Press 1983

21. Matsumoto, H., Nakano, Y., Umezawa, H.: Renormalization group at finite temperature. Phys. Rev. D29, 1116-1124 (1984)

22. Matsumoto, H., Ojima, I., Umezawa, H.: Perturbation and renormalization in thermo field dynamics. Ann. Phys. (N.Y.) 152, 348-375 (1984)

23. Maurin, K.: Analysis. Part I. Dordrecht: Reidel 1976

24. Marichev, O.I.: Handbook of integral transforms of higher transcendental functions. Chicester: Ellis Horwood 1983

25. Mumford, D.: Tata lectures on theta, Vol. 1. Boston: Birkhäuser 1983

26. Nakanishi, N.: Graph theory and Feynman integrals. New York, San Fransisco: Gordon and Breach 1971

27. Oberhettinger, F.: Tables of Mellin transforms. Berlin, Heidelberg, New York: Springer 1974

28. Speer, E.R.: Generalized Feynman amplitudes. Princeton: Princeton University Press 1969

29. Speer, E.R.: Analytic renormalization. J. Math. Phys. 9, 1404-1410 (1968)

30. Zimmermann, W.: The power counting theorem for Feynman integrals with massless propagators. In: Renormalization theory. Velo, G., Wightman, A.S. (eds.). Dordrecht: Reidel 1976

Communicated by K. Gawedzki

Received January 17, 1989 